\title{
Algunas notas sobre los Modelos de Desarrollo Regional en el ámbito del Medio Ambiente
}

\author{
José Antonio SoTELO NAVALPOTRO \\ Instituto Universitario de Ciencias Ambientales (IUCA/UCM) \\ jasotelo@ucm.es
}

Recibido: 17 de enero de 2013

Enviado a evaluar: 22 de enero de 2013

Aceptado: 15 de abril de 2013

\section{PRIMERA APROXIMACIÓN}

A la hora de interpretar los modelos de desarrollo, en el ámbito del medio ambiente y del territorio, puede ser de gran interés plantear una metodología que cumpla con la doble función de ser un instrumento de interpretación de los modelos aceptados hasta los momentos presentes, como para servir de base a otros que se planteen en un futuro próximo. $\mathrm{Y}$ es que la verdadera discusión sobre el medio ambiente puede resultar muy compleja, ya que cada uno tenemos sentimientos muy arraigados sobre el tema. Pero también es cierto que, incluso para los ambientalistas, resulta imprescindible que sepamos priorizar esfuerzos en campos muy diferentes; como la salud, la educación, las infraestructuras o la defensa, además del medio ambiente. Sin lugar a dudas, es en este ámbito en el que deben plantearse los nuevos modelos de desarrollo regional, cuya interpretación suele realizarse distinguiendo: "Los enfoques económicos tradicionales" y "los aspectos espaciales de las teorías de desarrollo económico. Respecto del primer apartado, señalar que la creciente atención que suscitan fenómenos como los que estamos tratando, ha generado una ingente obra científica elaborada en torno al crecimiento económico y al desarrollo, las causas y los orígenes del progreso socioeconómico no dejan de constituir un misterio. Muchos y diversos han sido los factores ensalzados como promotores del desarrollo. Sin embargo, la mayoría de los enfoques pecan por defecto al limitarse al estudio de uno sólo (o a lo suma dos) de sus elementos constituyentes. No en vano buena parte de las teorías económicas del crecimiento y del desarrollo han sido concebidas a partir de un sólo componente; de hecho, la mayoría de los modelos tradicionales de desarrollo tienden a reducir la complejidad de social a su mínima expresión.

Además con harta frecuencia la dimensión espacial del fenómeno ha sido obviada o camuflada. El espacio ha quedado reducido, según el caso, a la más completo ignorancia o a la consideración de mero contenedor de elementos y procesos, frente a la idea de espacio como filtro adaptador de los procesos sociales o la realidad local. De hecho, la concepción del espacio bajo una óptica global, del medio espacial como 
un elemento básico en la transformación de procesos de origen externo, así como la idea de espacio como "moldeador" de la vida social han sido cuestiones extrañas al concepto imperante de desarrollo.

Entre "las teorías económicas tradicionales de desarrollo" podemos apuntar que, a pesar de que la idea de progreso y de crecimiento económico aparece en distintas fases del pensamiento de Smith, Ricardo y Engels y Marx, ha sido recientemente cuando los enigmas del desarrollo y del crecimiento han comenzado a atraer de manera notable el interés de los científicos sociales. De esta manera hemos podido asistir al nacimiento de una gran cantidad de modelos de desarrollo a lo largo del siglo XX. Escuelas, teorías y modelos como el estructuralista, el de crecimiento equilibrado, el de crecimiento desequilibrado, el del bajo nivel de equilibrio, el de la causalidad cumulativa circular, el de la dominación o dependencia, todos ellos intentando encontrar explicación al atraso económico. Estos enfoques han encontrado sus bases filosóficas y económicas en los paradigmas neoclásico, neomarxista,...

"Grosso modo", las teorías de desarrollo suelen agruparse en dos grandes apartados:

En primer lugar, encontramos aquellas teorías que propugnan la libre acción de las fuerzas del mercado; en segundo lugar, las denominadas teorías intervencionistas, en las que a la par se diferencian las teorías de equilibrio y las teorías de desequilibrio. Todas ellas, con mayor o menor fortuna, han proclamado de manera más o menos explícita, la universalidad y certeza de sus fundamentos, propugnando múltiples medidas para aliviar los desequilibrios económicos. Y, sin embargo, las desigualdades en los niveles espaciales de desarrollo, tanto a nivel mundial como a escala regional, no sólo no se han reducido, sino que se han mantenido e incluso han seguido creciendo en algunas áreas durante las últimas décadas. De hecho, la génesis y expansión de los desequilibrios sigue constituyendo un enigma.

Entre "los aspectos espaciales de las teorías de desarrollo" debemos decir que a pesar de que las teorías económicas de desarrollo fueron elaboradas sobre una base "aespacial" esto no quiere decir que sean territorialmente neutras. Cada una de las racionalizaciones sobre la génesis y evolución de los desequilibrios y cada interpretación de las posibles soluciones al problema lleva implícita la configuración de un modelo de desarrollo territorial. Por tanto, son diversas las implicaciones territoriales que se pueden extraer de las corrientes de desarrollo citadas anteriormente, destacando los modelos de Hirschman, Rostow, Perroux, Myrdal, Hágerstrand,...

Hemos de ser conscientes, no obstante, que de cualquier clasificación que se haga de la vida económica en períodos o sistemas hay que decir, que para la interpretación de los modelos de desarrollo regional, no significa que en cada uno de ellos se den de una manera única y exclusiva las notas que los caracterizan. Con Max Weber hab que decir que más que realidades empíricas, representan tipos abstractos de organización económica que sirven para valorar y clasificar la realidad más o menos histórica de un desarrollo, siendo conscientes que en la actualidad deberíamos realizar un número elevado de combinaciones para acercarnos a la realidad vigente en los distintos Estados. 


\section{ASPECTOS DIACRÓNICOS EN LA INVESTIGACIÓN DE LOS MODELOS DE DESARROLLO REGIONAL}

Frente a la tradición francesa o alemana, en la investigación sobre el desarrollo regional destaca el que podríamos calificar como contexto anglosajón, encontrándonos con el arquetipo: “¿Ciencia regional vs. Economía política regional?”. En ellos pervive la escisión entre "planificación socioeconómica" (socioeconomic planners) y "planificación física" (environmental planners y/o physical designers). La práctica del desarrollo regional podemos observarla en los trabajos de Friedmann (1979); se puede hablar de los modelos de desarrollo regional que se alimentan tanto de teorías de los procesos de planificación como de las teorías sustantivas del desarrollo regional. La primera concuerda con lo que podríamos llamar "teoría del desarrollo", y discute sobre si la planificación debe ser global o incrementalista, centralizada o descentralizada, tecnocrática o participativa, a corto o a largo plazo,... Por otra parte, la teoría sustantiva de la planificación regional incluye la revisión de las diferentes propuestas de geógrafos, economistas, sociólogos,..., en relación al territorio, a las políticas y estrategías concretas que se debieran seguir para afectar desde los modelos de desarrollo al futuro de una región. En todos los casos, las distintas propuestas estarán influidas por la ideología, que condicionará tanto las propuestas concretas de intervención como los resultados que eventualmente se obtengan (Curbelo, J.L. "et alia". 1989).

Desde una perspectiva epistemológica, los estudios e investigaciones regionales oscilan desde mediados de los setenta en un difícil equilibrio entre dos paradigmas igualmente legitimados, desde perspectivas académicas, aunque no tanto en la práctica, como son la "Ciencia Regional" y la "Economía Política Regional"; somos conscientes de que estas denominaciones son un tanto arbitrarias por cuanto es difícil encontrar en la actualidad representantes puros de ambos, dado que los primeros estudios regionales (lsard), que bebían de las fuentes de la economía neoclásica y keynesiana, y la geografía funcionalista (Berry), están siendo contestados por enfoques que reciben sus influencias intelectuales de las formulaciones neomarxistas del espacio, el desarrollo desigual, los movimientos sociales, y el Estado (Castells, Giddens, Harvey...); el análisis de los procesos de urbanización, la planificación y las burocracias de Weber, Mannhein, Veblen (Friedmann, Weaver, Szelenyi); y las aportaciones de las teorías de la crisis y los ciclos económicos de Schumpeter, Kondratieff, etc. (Hall, Markusen, Freeman).

Ambos paradigmas se nos presentan lejos de conformar cuerpos unificados de principios, ideas, métodos o políticas; tanto es así que no podemos afirmar que uno de ellos sea "superior" al otro. Sin embargo, debemos señalar que mientras la mayoría de los que trabajan los modelos de desarrollo en el ámbito de la economía política regional son críticos de las asignaciones del mercado e intentan ligar el análisis económico con una "praxis" encaminada a cambiar las relaciones sociales de producción, los científicos regionales no cuestionan la estructura de clases existentes, sino que intentan dar soluciones para mejorar la eficiencia del mecanismo de mercado y corregir sus imperfecciones (Curbelo, J.L. "et alía". 1989). 
Siguiendo el estudio citado del profesor J. L. Curbelo (1989), los orígenes del paradigma los podemos encontrar en los trabajos deJ. Von Thiinen, A.Weber, A.Lósch, B. Ohlin,..., destacando los estudios de W. lsard. Basada en un contexto neoclásico la "teoría económica regional incluye el concepto de espacio bajo la forma dual de distancia (transporte) y suelo, que son "inputs" y componentes de la demanda de la empresa y el consumidor racional. Así, la interacción de las fuerzas del mercado da forma al sistema regional, siendo el desarrollo una consecuencia del "libre" fluir de los recursos de acuerdo a criterios de optimización.

Apartándose del modelo neoclásico simple, diversos estudiosos de formación keynesiana tuvo como consecuencia la creación de modelos como el de causación cumulativa y los denominados polos de crecimiento; estos enfoques vistos hasta ahora -e independientemente de su origen neoclásico o keynesiano- tienen en común el que ponen especial énfasis en el lado de la oferta del proceso de crecimiento, siendo la diferencia entre el modelo neoclásico puro y el modelo de causación cumulativa, eminentemente teórico. La interpretación que de las teorías de Keynes se ha realizado desde la Ciencia Regional se concreta en la idea de que las actividades exportadoras (los denominados sectores básicos) conforman un componente de la demanda, que además capto riqueza externa que se dirige hacia la economía regional, o en la hipótesis de que los problemas regionales se derivan de las faltas de inversiones, siendo necesarios, por tanto, incentivos e inversiones públicas dirigidas a incrementar el atractivo de la región para el capital externo (a esta misma conclusión, aunque desde posiciones metodológicas diferentes han llegado algunos de los mentores de la teoría del desarrollo endógeno).

Pese a todo, con haber sido importantes las aportaciones teóricas de la "Ciencia Regional", y haber tenido un peso notable en las políticas de desarrollo puestas en práctica hasta finales de los años setenta, en la actualidad sus preocupaciones y aportaciones se dirigen más a la confección de "métodos de análisis" (v.gr.: técnica del "shift-share", análisis "input-output", métodos "RAS" y de entropía, "matrices de contabilidad social", control presupuestario y análisis de proyectos -coste-beneficio-). Eso sí, los métodos cuantitativos y las técnicas de análisis señaladas, de orientación eminentemente aplicada, se han visto complementados por enfoques cualitativos que aportan una visión más humana de los problemas que se intentan solucionar.

Dentro de este paradigma se incluyen dos escuelas que cada vez tienen más importancia en las investigaciones regionales, a saber: "los Estudios regionales" y la "Economía Política Espacial”.

El primero de ellos -"los Estudios Regionales" aparece en el Reino Unido en los prolegómenos de los setenta, y tiene su principal mentor en Peter Hall, encontrando notables seguidores en diversas agencias internacionales de desarrollo (IIPES-CEPAL, UNCR, ...), así como en estudiosos como Teitz, Friedmann, Lewis Munford, H. Odum, Stóhr, Sergio Boisier. Esta escuela ha cuestionado las bases sociales y epistemológicas de la Ciencia Regional, criticando sobre todo su falsa neutralidad y su concepción naturalista de las ciencias sociales; rechazan así el "monopolio economicista" que caracterizó al anterior paradigma, conceptualizando los estudios regionales a partir de las cuatro disciplinas enumeradas por Kukliski: economía, sociología, ecodesarrollo y 
politología. Igualmente, esta "corriente" matizo la importancia de los métodos analíticos, proponiendo metodologías de análisis cualitativo (sirvan como ejemplo, la"observación participativa" o la "fenomenología").

La segunda tendencia es la "Economía Política Espacial" que arranca, a partir de la severa critico que se realizó de la intervención del Estado del bienestar, cuenta con investigaciones como los de Holland, Massey, Hudson, Lewis, Marcusen Wolker, publicadas en revistas como "International Journal of Urban and Regional Research" "Environnment and Plannig", "Landscape and urban planning. Los autores de esto tradición buscan entender lo que se conoce como modo de producción capitalista en el que coexisten sujetos sociales que desempeñan funciones distintas en la producción y uso del "producto social"; prestan atención no tanto a los decisiones de los sujetos, como en la Ciencia Regional, sino a las condiciones de equilibrio y desarrollo de todo el sistema. Así, a diferencia de los científicos neoclásicos, paro quienes la economía es lo ciencia de la elección humano en condiciones de escasez, los políticoseconomistas se centran en las leyes de movimiento del sistema. El espacio no es un epifenómeno como lo es para la Ciencia Regional, sino un elemento central al proceso de acumulación, estando la Economía Político Regional preocupado por el estudio de los mecanismos por los cuales el proceso de acumulación genero desarrollo desigual en el espacio, y los efectos de tales desequilibrios en el desarrollo de la formación social nacional y de las distintas áreas.

Este complejo entramado de enfoques, análisis, debates y temas de estudio que se generan dentro de este paradigma puede resumirse en cuatro amplios epígrafes:

En primer lugar, el estudio de las "leyes de movimiento" del sistema capitalista, la división internacional del trabajo, y los efectos regionales de la reestructuración capitalista y la salido de la crisis.

En segundo lugar, las manifestaciones de las leyes del movimiento capitalista en los sectores económicos y los distintos regiones.

En tercer lugar, el análisis del Estado y sus intervenciones en los niveles local, regional y nacional.

En cuarto lugar, y relacionado con los epígrafes anteriores, el estudio del regionalismo y los movimientos sociales regionales.

Lo hasta aquí expuesto debería verse enriquecido por las oportaciones de la Geografía, sin embargo, "esta ciencia siempre en crisis", no ha sabido desde su corpus teórico dar respuesta a los problemas con que se han enfrentado ciencias como la Economía, o la Sociología. De una u otra forma, tanto la "literatura" como "las investigaciones" citadas, pueden ayudarnos a comprender e interpretar mejor los susodichos modelos de desarrollo regional, coadyuvando la formalización de una metodología de investigación como la presente. 


\section{EL COMPLEJO MUNDO DE LA INTERPRETACIÓN DE LOS MODELOS, UN PUNTO Y SEGUIDO}

En la actualidad la aldea global ha sido sustituida por el mercado global, dando lugar al tratado tema de la globalización, que, en su actual modalidad neoliberal, tiene carácter excluyente tanto de continentes enteros, los más subdesarrollados, como de sectores cada vez más amplios de los países desarrollados, los que no se adaptan a la permanente renovación tecnológica. Así, podemos plantearnos el siguiente interrogante: ¿por qué los modelos de desarrollo regional? "Es incontestable", afirma Enzensberger, "que el mercado mundial, desde que dejó de ser una visión lejana y se convirtió en realidad global, fabrica cada vez menos ganadores y más perdedores, y eso no en el Tercer Mundo o en el Segundo, sino en los altos centros del capitalismo. Allá son países y continentes enteros los que se ven abandonados y excluidos de los intercambios; aquí son sectores cada vez más grandes de la población los que, en la competencia cada día más grande por las calificaciones, no pueden seguir y caen".

Nadie puede negar, como hemos señalado, que la globalización de la economía reporta muy notables beneficios. Pero no se reparten equitativamente; muy al contrario los países desarrollados cada vez se benefician más que los subdesarrollados, e incluso, en un mismo país, los ricos logran más ganancias que los más pobres. A lo largo de los próximos años, la liberalización del comercio reportará entre 212.000 y 510.000 millones de dólares. Sin embargo, los 48 países más subdesarrollados tendrán unas pérdidas anuales de 600 millones en material comerciable, y los del África subsahariana, alcanzarán pérdidas que superarán los 1.200 millones. También ha crecido el producto, el PIB mundial, en términos globales. Sin embargo, el de 44 naciones subdesarrolladas se ha visto seriamente mermado (Tamayo-Acosta, J.J. 1999).

El informe sobre la "Pobreza y el desarrollo en el mundo, en 2008" se centraba en el consumo, abunda en el carácter desigual y excluyente de la globalización. El consumo de bienes y servicios llegará a superar los 24 billones de dólares -seis veces la cifra de 1975- . Globalmente, se ha llegado a un nivel de consumo de alimentos, energía, educación, transporte, comunicación y ocio nunca alcanzado. Ahora bien, de nuevo los niveles de consumo no son ni mucho menos homogéneos, ya que el $20 \%$ de la población mundial realiza el $86 \%$ de los gastos en consumo personal.

Desde esta perspectiva, los modelos de desarrollo regional pueden servir al doble reto de aproximar al desarrollo los países subdesarrollados, y encontrar un equilibrio entre globalización y regionalización. En este sentido, deben convertirse en un coadyuvante más, al servicio de los Estados, regiones,..., para superar las diferencias SurNorte, colocar la economía al servicio del desarrollo integral de las personas y los pueblos, denunciar el carácter idolátrico del capital, defender la democratización de los Estados en torno a los valores comunitarios,... (Tamayo-Acosta, J.J. 1999).

Dar respuesta al interrogante anterior, nos obliga a plantearnos la base conceptual del "subdesarrollo", o por alusión su antónimo "desarrollo". La revisión de la ya abundante literatura especializada conduce a un sinnúmero de definiciones que más que enmarcar el fenómeno lo que hacen es caracterizar o tipificar alguna o algunas de sus 
manifestaciones, las más de las veces con criterios productivistas ajenos, en general, a la inseparable dimensión social que acompaña a las variables económicas.

A título ilustrativo se han seleccionado algunas aproximaciones de estudiosos que a lo largo del último medio siglo, se han preocupado del tema desde diferentes enfoques ideológicos. Así $\mathrm{H}$. Myint pasa revista a una serie de criterios utilizados para encuadrar el subdesarrollo comenzando por aquellos que se apoyan en el "nivel de renta por habitante" o en su tasa de crecimiento, lo que permite distinguir a los países con rentas y ritmos de crecimiento estancados o declinantes de aquellos otros con mayor dinamismo positivo, los industrializados. El desfase creciente (widening gap) entre ambos grupos de países se constituye en criterio diferencial, válido para una clasificación pero, a nuestro juicio, no para la conceptualización. Por su parte, Marrama analiza algunos ensayos de definición señalando, obviamente, la imprecisión de la que considera "un estado de subdesarrollo allí donde la población es escasa con relación a los recursos naturales disponibles" y valorando en mayor medida la de Viner, que califica como tales "los países que tienen buenas perspectivas potenciales para utilizar más capital o más mano de obra o más recursos naturales disponibles, o estos tres factores con objeto de elevar el nivel de vida de su población actual o, si su renta per cápita ya es alta, sostener una población más numerosa sin que disminuya su nivel de vida". Una más en esta línea es la de Lange, para quien "una economía subdesarrollada es una economía en la que el equipo de capital existente no es suficiente para el pleno empleo de la fuerza de trabajo disponible, de acuerdo con las técnicas modernas de producción". Pero esta aportación, de notable valor tecnoeconómico, pone el énfasis, al igual que las anteriores, en aspectos cuantitativos -potencial productivo escaso u ocioso-, si bien ya recoge la dimensión social, el paro o el subempleo; sin embargo, limita el fenómeno a una visión estática e impregnada en un cierto matiz "economicista", caracteres ambos que no son los que mejor pueden reflejar la globalidad y "dinamicidad" que el concepto encierra (Paz, M.A de., 1998).

Como escribió el admirado y añorado profesor José Manuel Casas Torres (1982), es obvio que todos intuimos lo que es el subdesarrollo, pero nadie -acabamos de apuntarlo- lo ha definido con precisión. Está claro que no es sólo una situación económica deprimida, y mucho menos un estadio cultural atrasado, pues dentro de la extensa gama de países incluidos en esta categoría, los hay con culturas milenarias riquísimas, y tampoco parece que todos los países desarrollados puedan darles lecciones en este terreno.

Es indudable, en cambio, que la condición de país subdesarrollado lleva consigo una situación de dependencia -política, económica, técnica,...- de otra potencia o de un grupo económico extranjero; supone también, en muchas ocasiones, una falta de participación política de su población en las tareas de gobierno, unas veces porque carece de preparación suficiente y otras porque se le impone una forma de administración sea paternalista o tiránica, que la excluye de ella. El subdesarrollo lleva implícito, desde luego, un nivel de vida extremadamente bajo, un predominio de población rural, un alto grado de analfabetismo, de paro total y paro encubierto; hambre y malnutrición crónicas, por tanto, a veces en grado muy difícil de imaginar, que hacen casi imposible el trabajo intenso y continuado: alta mortalidad infantil, morbilidad muy acusada, mala situación 
de la mujer -discriminada y considerada inferior- y, en ciertos países -como es de sobra conocido-, una administración incompetente, arbitraria, venal y corrompida.

Las nociones de desarrollo y subdesarrollo corresponden a la óptica occidental, materialista -trátese de capitalismo y comunismo-, que da prioridad a los temas de carácter económico. Desde este punto de vista la diferencia entre unos y otros países es palmaria e innegable. Pero, desde el lado de los países subdesarrollados, ¿podemos decir que comparten los puntos de vista de la población de los países industriales? Indudablemente, no se puede contestar de un modo simplista, pero puede creerse que una buena parte de sus habitantes miran las cosas y la vida con ojos muy distintos. Insistimos una y mil veces en que no es sólo lo económico lo que caracteriza el subdesarrollo de los pueblos y las regiones, aunque quizá, a pesar de sus enormes dificultades, lo económico es lo más fácil de detectar y medir.

El tema del subdesarrollo es, ante todo y esencialmente, profundamente humano: tres cuartas partes de la humanidad padecen sus consecuencias, y eso se refleja en todos sus aspectos demográficos. Por todos los lados del análisis de las características de la población mundial -tasas de natalidad y mortalidad, índices de crecimiento, estructura por edades, composición profesional de la población, esperanza de vida, condiciones de la mujer, grado de instrucción, renta per cápita,...- encontraremos -con todas las gradaciones y matices que dan las diferencias entre las poblaciones de los países ricos y las poblaciones de los países pobres, entre los países desarrollados y los subdesarrollados. Es lógico -lo contrario sería imperdonable- que el interés humano del tema -es la vida de millones de otros hombres lo que está en juego- atraiga no sólo la atención de los científicos, resultando muy doloroso comprobar -aunque nadie que conozca un poco la historia puede sorprenderse- cómo las grandes potencias emplean a estos países como peones, y cabezas de puente, en el trágico juego de la estrategia universal, condicionando su ayuda a que se muevan según su conveniencia.

\section{BIBLIOGRAFÍA}

BOISIER, S. (1997). En busca del esquivo desarrollo regional: entre la caja negra y el proyecto político, Ciudad y Territorio, Estudios Territoriales, XXIX, 112, Pp. 379-397.

BRUNDTLAND, G. R. (1988). Our Common Future. The Report of the Wold Commission on Environment and Development. Oxford University Press.

CABERO DIÉGUEZ, V. (1994). Contribución para la definición de una estrategia de intervención y la promoción

CABERO DIÉGUEZ, Vi (1996). Los espacios naturales protegidos: el dilema entre conservación y el desarrollo local, en Dinamismos socio-económicos e (re) organizaçao territorial: procesos de urbanizaçao e de reestructuraçao productiva, Instituto de Estudios Geográficos, Universidad de Coimbra, pp. 501-510.

CABERO DIÉGUEZ, V. (2001). Espacios naturales protegidos y conservación del medio, en GIL OLCINA, A y Gómez Mendoza, J. (coord.). Geografía de España, Barcelona, Ed. Ariel, pp. 207-221. 
CASAS TORRES, J. M. (1982). Población, desarrollo y calidad de vida. Madrid. Rialp. 338 págs.

CASTELLS, M. et al. (1986). Nuevas tecnologías, Economía y Sociedad en España, Madrid, Alianza Edit. 2 vols.

CASTELLS, M. (1999). El mundo según flavos, El País, 12 de febrero, pág. 18.

CEE. Primer Programa Europeo contra la pobreza (19 75-1980).

CEE. Segundo Programa Europeo contra la pobreza (1984-1988).

CEE. Tercer Programa Europeo contra la pobreza (1990-1995).

CLUB DE BRUXELLES (1992). L'Environnement dans le Marché unique European, Vol. 1, Pp. 61-63.

CORTÉS, R. (1996). El Programa Español de Desarrollo y Diversificación Económica de Zonas Rurales Objetivo 1, Baetica, vol. 18, Pp. 63/72.

CURBELO, J.L. et alii. (1989). Los estudios regionales en el contexto anglosajón: ¿"Ciencia regional” vs. “Economía política regional”? Estudios Regionales, $\mathrm{n}^{\circ}$ 23., pp. 15-27.

FURIÓ, E. (1994). El desarrollo económico endógeno y local: reflexiones sobre su enfoque interpretativo, Estudios Regionales, n.0 40, Pp. 97-112.

GARCÍA ALVARADO, J.M. (1997). Propuesta teórico-metodológica para la valoración de la calidad urbano ambiental, Anales de Geografía de la 11CM,

n.0 17, Pp. 11-26.

GARCÍA ALVARADO, J.M. (2000). El modelo metropolitano madrileño: enjuiciamiento desde la perspectiva del V Programa de Acción de CE. En materia de Medio Ambiente. Apuntes metodológicos, Observatorio Medioambiental, n.0 3, pp. 135-158.

GARCÍA BALLESTEROS, A. (1998). Nuevos espacios del consumo y exclusión social, Anales de Geografía de la 11CM, n.0 18, pp. 47-53.

GARCÍA DE ENTERRIA, E. (1997). Economía y secularización de la política, Madrid, $A B C, 27$ de noviembre.

LEE, N. (1991). Quality control in the EIA process. EIA Newsletter 6:22-23.

LILLIAN, T et alii. (1977). Urbanization -..-Industrialization andthe Theory of Demographic Transition. Pacific Sociological Review, vol. 20. nº 1, pp. 1 13-134.

PAZ, M.A. (1998). Economía mundial. Madrid, Edt. Pirámide, 605 págs.

RAMOS, A. et alii. (1979). Planificación física y ecología. Modelos y métodos. Madrid. Ed. E.M.E.S.A.

RAMOS, A. (edt.) (1987). Diccionario de la naturaleza. Hombre, ecología y paisaje. Madrid. Espasa-Calpe.

RAMOS, A. (coordi.) (1990). Medio Ambiente y crecimiento económicos. Revista del Instituto de Estudios Económicos, n.0 2.

SOTELO NAVALPOTRO, J. A. (1999). Modelos de Organización y Desarrollo

Regional, Madrid, IUCA, $195 \mathrm{pp}$.

SOTELO NAVALPOTRO, J. A. (2000a). Regional Development Models, Oxford University Press. 
SOTELO NAVALPOTRO, J. A. (2000b). Medio Ambiente y Desarrollo en España en los prolegómenos del siglo XXI: Las Políticas Medioambientales de la UE, Observatorio Medioambiental, n.0 3, pp. 34 1-397.

STÖHR, W. B. (1986). La politique japonaise des technolopes: Innovation Technologique et industrielle, en Federwich, J. et alii. Technologie nouvelle et ruptures regionales, Paris, Económica, PP. 123/139.

SOLOZÁBAL, J. M. (1989) Curso de Economía, Bilbao, Ed. Deusto.

TAMAYO-ACOSTA, J.J. (1999). El cristianismo en tiempos de globalización, El País, 4 de enero, pág. 12. 